\title{
POLITICA MONETARIA DE ECONOMIAS ABIERTAS: EL ROL DEL TIPO DE CAMBIO EN MEXICO
}

\author{
MONETARY POLICY IN OPEN ECONOMIES: \\ THE ROLE OF THE EXCHANGE RATE IN MEXICO
}

\section{OSCAR PEREZ-LAURRABAQUIO*}

\begin{abstract}
This paper analyzes the role of the exchange rate in Mexico during the inflation targeting regime. The framework is based on the New Keynesian Macroeconomics, and the empirical evidence in the estimation of a Vector Error Correction model and the statistical analysis of the inflation targeting in Mexico and Latin America. The results describe to the Mexico's monetary policy as a hybrid system, where the nominal interest rate (policy instrument) and appreciations of the real exchange rate (intermediate instrument), have as their primary objective, the inflation stabilization within a numerical goal.
\end{abstract}

Keywords: Time series models, monetary policy, exchange policy.

JEL Classification: C32, E52, F31.

\section{Resumen}

Este trabajo analiza el rol del tipo de cambio en México durante el esquema de metas de inflación. El marco de referencia lo sustenta la Nueva Macroeconomía Keynesiana, y la evidencia empírica la estimación de un

* E-mail: laurrabaquio@comunidad.unam.mx

Agradezco los valiosos comentarios de Violeta Rodríguez del Villar y José García-Solanes. Se aplica el descargo usual de los errores. 
modelo de Vector de Corrección del Error y el análisis estadístico de las metas de inflación en México y América Latina. Los resultados obtenidos describen a la política monetaria de México como un sistema híbrido, donde la tasa de interés nominal (instrumento de política) y apreciaciones del tipo de cambio real (instrumento intermedio), tienen como primordial objetivo, la estabilización de la inflación dentro de una meta numérica.

Palabras clave: Modelos de series de tiempo, política monetaria, política cambiaria.

Clasificación JEL: C32, E52, F31.

\section{INTRODUCCION}

Durante las dos últimas décadas del siglo XX, el uso potencial de las prácticas keynesianas y la aplicación sin precedentes de la hipótesis de expectativas racionales, supusieron (en detrimento del modelo IS-LM) el surgimiento de la Nueva Macroeconomía Keynesiana (NMK) como paradigma dominante de la macroeconomía moderna (Clarida, Galí y Gertler, 1999). Bajo la aceptación de rigideces nominales en los precios (Calvo, 1983), en este consenso el nivel de inflación se determina por la política monetaria, es decir, la tasa de interés (Blinder, 1999); y los valores de equilibrio de las variables reales, se afectan a corto plazo por su mecanismo de ajuste (Clarida, Galí y Gertler, 2001). Consecuentemente, el modelo eleva el control de los precios al rango de objetivo prioritario del banco central (Bernanke y Mishkin, 1997; Mishkin, 2000), en el entendido de que una baja y estable tasa de inflación es consistente con una tasa natural de desempleo (hipótesis NAIRU) (Arestis y Sawyer, 2003).

En su análisis técnico, la fijación de metas cuantitativas de inflación como marco operativo del banco central, constituye la piedra angular de la nueva macroeconomía. De acuerdo con su funcionamiento, el régimen de metas de inflación presupone el uso de una regla monetaria de tasa de interés (Ball, 1997; Svensson, 1996; Taylor, 1993, 1998), como instrumento fundamental para estabilizar el nivel de precios (dentro de un objetivo/rango numérico). Además de que la política fiscal se concibe como un instrumento macroeconómico ineficiente (Mishkin, 2000, 2004), el régimen cambiario debe ser de libre flotación (Svensson, 2001); ya que una paridad flexible al no intervenir en la política monetaria, le permite a la autoridad central el oportuno cumplimiento de sus objetivos (Savastano, Masson y Sharma, 1997).

La primera economía en adoptar esta estrategia fue Nueva Zelanda en 1990, al fijar un rango de inflación de 0 a 2\%, que habría de mantenerse hasta el 31 de agosto de 1993 (RBNZ, 1990). Desde entonces, un número creciente de países industrializados y en vías de desarrollo, han visto con buenos ojos la adopción de las metas de inflación como su esquema predilecto de política monetaria (Hammond, 2012; Schmidt-Hebbel 
y Carrasco, 2016). En México, la autonomía constitucional de su banca central y libre flotación del tipo de cambio (ambos en 1994), permite que en enero de 1999 se adopte (explícitamente) una meta de inflación del 13\%, siendo hasta el 2002, cuando se llega al actual objetivo de $3 \%+/-1 \%$ (medido a través del cambio en el Indice Nacional de Precios al Consumidor, INPC) (Pérez-Laurrabaquio, 2014).

La relación entre tipo de cambio y tasa de inflación, se suscribe en que una moneda depreciada mejora los términos de intercambio de la economía doméstica a costa de un aumento en el nivel general de precios (Pontines y Siregar, 2012, 2015), mientras que una moneda apreciada tiene el efecto inverso en la economía (Libman, 2018). La evidencia empírica del trabajo toma como antecedente inmediato las investigaciones que analizan la relación entre tipo de cambio apreciado y baja tasa de inflación (Barbosa-Filho, 2015; Ros, 2015), a través del análisis de reglas monetarias para economías abiertas (Cermeño, Villagómez y Orellana Polo, 2012; de Mello y Moccero, 2011; Ramos-Francia y Torres, 2005). En nuestro caso, los altos niveles de inflación de 1990 y principios de siglo, así como la crisis de balanza de pagos de 1994, ponen en el centro de la política monetaria el rol del tipo de cambio durante el esquema de metas de inflación.

Dado el alto grado de apertura comercial y lo poco desarrollado que se encuentran los mercados de crédito en los países emergentes (de Paula y Ferrari-Filho, 2010), se vuelve muy tentador la subordinación del tipo de cambio a los propósitos de la política monetaria (Ghosh, Ostry y Chamon, 2016). Con la eminente contradicción de política económica que ello implica, en los siguientes apartados se investiga "el rol del tipo de cambio real en México durante el esquema de metas de inflación”. Los resultados obtenidos describen a la política monetaria del país como un sistema híbrido, donde la tasa de interés nominal (instrumento de política) y apreciaciones del tipo de cambio real (instrumento intermedio), tienen como primordial objetivo, la estabilización de la inflación dentro de una meta numérica.

Posterior a esta sección introductoria, la estructura del trabajo es la siguiente. La sección 2 la componen los supuestos, control robusto y solución del modelo teórico. La sección 3 la revisión de la literatura, estimaciones econométricas para la economía mexicana, y la evidencia estadística del desempeño de las metas de inflación en México y América Latina. En las conclusiones se presentan las aportaciones de la investigación, y finalmente la bibliografía y anexo estadístico.

\section{MODELO TEORICO}

\subsection{Supuestos}

Similar a Clarida, Galí y Gertler (2001) y Leitemo y Söderström (2008), el modelo supone dos tipos de países: uno pequeño (economía doméstica) que no influye en los niveles de producción, precios y tasa de interés del exterior; y uno grande (economía 
foránea) donde ocurre lo contrario. Los agentes (hogares y firmas) de ambos países, intercambian libremente activos financieros y bienes de consumo, y las rigideces nominales en los precios, permiten un trade-off inflación-producto a corto plazo. Preservando su abstracción, todas las variables se expresan como la desviación porcentual de sus niveles de equilibrio a largo plazo.

En lo anterior, la política monetaria de la NMK para una economía doméstica representativa, se estiliza con las siguientes ecuaciones:

$$
\begin{gathered}
\tilde{\pi}_{t}=\alpha E_{t} \tilde{\pi}_{t+1}+\beta \tilde{y}_{t}+\gamma e_{t}+\varepsilon_{t}^{\tilde{\pi}} \\
\tilde{y}_{t}=E_{t} \tilde{y}_{t+1}-\delta\left(i_{t}-E_{t} \pi_{t+1}\right)-\theta\left(E_{t} e_{t+1}-e_{t}\right)+\varepsilon_{t}^{\tilde{y}} \\
e_{t}=E_{t} e_{t+1}-\left(i_{t}-E_{t} \pi_{t+1}\right)+\left(i_{t}^{*}-E_{t} \pi_{t+1}^{*}\right)+\varepsilon_{t}^{e}
\end{gathered}
$$

Donde $\tilde{\pi}_{t}$ es la brecha de inflación, $\tilde{y}_{t}$ la brecha del producto, $e_{t}$ el tipo de cambio real, $i_{t}$ la tasa de interés nominal, $\pi_{t+1}$ la tasa de inflación del siguiente período, $\left(i_{t}-E_{t} \pi_{t+1}\right)$ la tasa de interés real $\left(r_{t}\right),\left(i_{t}^{*}-E_{t} \pi_{t+1}^{*}\right)$ la tasa de interés real foránea $\left(r_{t}^{*}\right), \mathrm{y}\left(E_{t} e_{t+1}-e_{t}\right)$ la tasa de variación esperada del tipo de cambio real. Asimismo, $E_{t}$ es el operador valor esperado, $\alpha, \beta, \gamma, \delta$ y $\theta$ parámetros positivos, y $\varepsilon_{t}^{j}$ shocks estacionarios (i.i.d).

La ecuación (1) es esencialmente una curva de Phillips, que relaciona la brecha de inflación con su valor esperado en $t+1$, la brecha del producto, el tipo de cambio real, y un shock de oferta $\left(\varepsilon_{t}^{\tilde{\pi}}\right)$ por variaciones de los costes de producción. La ecuación (2) es una curva IS para la brecha del producto, explicada por su valor esperado en $t+1$, la tasa de interés real, la tasa de variación esperada del tipo de cambio real, y un shock de demanda $\left(\varepsilon_{t}^{\tilde{y}}\right)$ por cambios imprevistos en la inversión y gasto público. La ecuación (3) describe la paridad descubierta de tasas de interés, asociando al tipo de cambio real con su valor esperado en $t+1$, la tasa de interés real doméstica, la tasa de interés real foránea, y un shock de tipo de cambio $\left(\varepsilon_{t}^{e}\right)$ que denota su prima de riesgo.

Respecto a la política monetaria, si los bancos centrales consideran que la tasa de interés "nominal" es un instrumento eficiente de su plan de trabajo (Taylor, 1993)1, se define una función de pérdida para sus objetivos de política:

1 Como la tasa de interés real es más una conjetura aritmética que una cantidad fija (al desconocerse el valor exacto de $\pi_{t+1}$ en $t$ ), las presiones de inflación se eliminan incrementando la tasa de interés nominal más que proporcionalmente al aumento de los precios (Taylor, op. cit.). De esta manera, el instrumento de política a considerar, corresponde a $i_{t}$ en sustitución de $r_{t}$. 


$$
\begin{gathered}
\min _{\left\{i_{t}\right\}} E_{t} \sum_{t=0}^{t=\infty}\left[\lambda_{\tilde{\pi}} \tilde{\pi}_{t}^{2}+\lambda_{\tilde{y}} \tilde{y}_{t}^{2}\right] \\
0<\lambda_{j} \leq 1
\end{gathered}
$$

Donde los valores no-negativos de $\lambda_{j}$ son la suma ponderada (parámetros de preferencia), que la autoridad central le da a la estabilización de la inflación versus la estabilización del producto. Por ejemplo, si $\lambda_{\tilde{\pi}}=\lambda_{\tilde{y}}=1$, los movimientos de ambas variables se ponderan con la misma importancia; si $\lambda_{\tilde{\pi}}>\lambda_{\tilde{y}}$, hay menos aversión hacia $\tilde{\pi}_{t}$ respecto de $\tilde{y}_{t}$; y lo contrario si $\lambda_{\tilde{\pi}}<\lambda_{\tilde{y}}$. Además, si $\lambda_{\tilde{\pi}}=1$ y $\lambda_{\tilde{y}} \approx 0$, la autoridad monetaria se rige por un estricto esquema de política: el control de la inflación como su única prioridad.

El Banco de México, al tener como primordial misión "la estabilidad del poder adquisitivo de la moneda nacional, fortaleciendo con ello la rectoría del desarrollo [...] que corresponde al Estado" (Cámara de Diputados, 1993: art. 28), las ponderaciones de sus objetivos se definen como $\lambda_{\tilde{\pi}}=1$ y $0<\lambda_{\tilde{y}}<1$; es decir, se supone un pleno compromiso por controlar el nivel general de precios, sin descartar por completo la importancia de la brecha del producto.

Para colapsar el modelo, bajo una perfecta movilidad de capitales en el mercado financiero global, la libre flotación del tipo de cambio permite el cumplimiento de la paridad descubierta de tasas de interés: la diferencia entre tipos de interés $\left(r_{t}-r_{t}^{*}\right)$ es igual a la tasa de variación esperada del tipo de cambio real $\left(E_{t} e_{t+1}-e_{t}\right)$. Sin embargo, la ya mencionada apertura comercial y precario desarrollo de los mercados de crédito en los países emergentes, convierten al tipo de cambio en una variable relevante del análisis monetario. Como una fehaciente explicación a la realidad de estos países, el régimen cambiario a discutir en adelante corresponde al de una flotación intervenida, donde los movimientos del tipo de cambio real y tasa de interés nominal, optimizan la función de pérdida del banco central a través de una regla de política monetaria.

\subsection{Control robusto}

Para construir la regla de política monetaria, las ecuaciones (1), (2) y (3) como restricciones de (4) (Leitemo y Söderström, 2008), forman el siguiente lagrangiano:

$$
\begin{aligned}
E_{t} \sum_{t=0}^{t=\infty}\{ & \tilde{\pi}_{t}^{2}+\lambda_{\tilde{y}} \tilde{y}_{t}^{2}-\mu_{t}^{\tilde{\pi}}\left[\tilde{\pi}_{t}-\alpha E_{t} \tilde{\pi}_{t+1}-\beta \tilde{y}_{t}-\gamma e_{t}-\varepsilon_{t}^{\tilde{\pi}}\right] \\
& -\mu_{t}^{\tilde{y}}\left[\tilde{y}_{t}-E_{t} \tilde{y}_{t+1}+\delta\left(i_{t}-E_{t} \pi_{t+1}\right)+\theta\left(E_{t} e_{t+1}-e_{t}\right)-\varepsilon_{t}^{\tilde{y}}\right] \\
& \left.-\mu_{t}^{e}\left[e_{t}-E_{t} e_{t+1}+\left(i_{t}-E_{t} \pi_{t+1}\right)-\left(i_{t}^{*}-E_{t} \pi_{t+1}^{*}\right)-\varepsilon_{t}^{e}\right]\right\}
\end{aligned}
$$


Suponiendo en el sistema un equilibrio discrecional ${ }^{2}$, se obtienen las condiciones de primer orden de la brecha de inflación, brecha del producto, tasa de interés nominal y tipo de cambio real:

$$
\begin{gathered}
\partial_{\tilde{\pi}_{t}}: \mu_{t}^{\tilde{\pi}}=2 \tilde{\pi}_{t} \\
\partial_{\tilde{y}_{t}}: \mu_{t}^{\tilde{y}}=2\left[\beta \tilde{\pi}_{t}+\lambda_{\tilde{y}} \tilde{y}_{t}\right] \\
\partial_{i_{t}}: \mu_{t}^{e}=-2 \delta\left[\beta \tilde{\pi}_{t}+\lambda_{\tilde{y}} \tilde{y}_{t}\right] \\
\partial_{e_{t}}: \mu_{t}^{e}=\gamma \mu_{t}^{\tilde{\pi}}+\theta \mu_{t}^{\tilde{y}}
\end{gathered}
$$

Para definir el rol del mercado cambiario (de flotación intervenida), al sustituir en (9) las condiciones (6), (7) y (8), se incluyen los movimientos del tipo de cambio al plan de trabajo del banco central:

$$
\begin{gathered}
\tilde{\pi}_{t}=-A^{-1} \tilde{y}_{t} \\
A^{-1} \equiv\left[\frac{\beta}{\lambda_{\tilde{y}}}+\frac{\gamma}{\lambda_{\tilde{y}}(\delta+\theta)}\right]>0
\end{gathered}
$$

Siendo los elementos fraccionados de $A^{-1}$, dos diferentes esquemas de política económica: (i) el uso exclusivo de la política monetaria en el control de la inflación y producto (economía cerrada); y (ii) la combinación óptima política monetaria-política cambiaria hacia el mismo fin (economía abierta).

En el primer esquema $\left[\beta / \lambda_{\tilde{y}}\right]$, el control de la brecha de inflación es directamente proporcional al valor de $\beta$ : un alto (o bajo) valor del coeficiente, supone un fuerte (o débil) control de la inflación vía la brecha del producto (curva de Phillips). Con $\lambda_{\tilde{y}}$, si su valor aumenta por la aversión del banco central hacia la brecha de inflación, existe un alto grado de compromiso en la estabilización del producto; pero si $\lambda_{\tilde{y}}$ disminuye

2 En Leitemo y Söderström, op. cit., la adopción de una política discrecional que no toma la persistencia de las variables como endógena, permite la solución del modelo analítico (principal contribución del trabajo). No obstante, una justificación de por qué se eliminan los valores futuros de la ecuación (5), tiene que ver con la idea de que las decisiones de política generalmente se toman "paso a paso" (esquema preventivo), evitando así la posibilidad de "quedar atrapado" en decisiones de larga duración (Blinder, 1999: 12-19). 
por las razones opuestas, la función de pérdida (ecuación 4) se inclina por un robusto control de los precios. En ambos casos, $\beta / \lambda_{\tilde{y}}$ representa el trade-off inflación-producto de una economía cerrada, donde una baja tasa de inflación requiere (propia natura) de una tasa de sacrificio en la brecha del producto.

El segundo esquema $\left[\gamma / \lambda_{\tilde{y}}(\delta+\theta)\right]$, expresa simultáneamente los canales de transmisión de las políticas monetaria y cambiaria. Con la primera de ellas (parámetro $\delta$ ), sigue prevaleciendo el modus operandi de una economía cerrada: una robusta (o laxa) tasa de interés, disminuye (o aumenta) la brecha del producto (curva IS), y en consecuencia estabiliza (o no) la brecha de inflación (curva de Phillips). Con la política cambiaria (parámetro $\theta$ ), un alza de la tasa de interés que aprecia el tipo de cambio por la atracción de flujos de capital del exterior (ecuación 3), disminuye la brecha de inflación de la curva de Phillips ante la caída de la brecha del producto de la curva IS; y una política monetaria expansiva que deprecia los términos de intercambio, tiene el resultado opuesto en la economía. Asimismo, al relacionarse el mecanismo de transmisión del parámetro $\gamma$ directamente con la brecha del producto (curva de Phillips), una apreciación cambiaria que abarata el precio de los bienes de consumo procedentes del exterior, estabiliza la brecha de inflación ante la caída del nivel general de precios.

Como ambos tipos de política necesitan la contribución de la tasa de interés para cumplir su cometido, el tipo de cambio real se considera un instrumento intermedio del banco central, y la tasa de interés nominal, el instrumento de política por excelencia.

\subsection{Solución}

Para formalizar las relaciones anteriores en una unívoca expresión, al sustituir en (2) a $\tilde{y}_{t}$ de la ecuación (10) y resolviendo para la tasa de interés:

$$
i_{t}=E_{t} \pi_{t+1}+\delta^{-1} A \tilde{\pi}_{t}-\delta^{-1} A E_{t} \tilde{\pi}_{t+1}-\delta^{-1} \theta\left(E_{t} e_{t+1}-e_{t}\right)+\delta^{-1} \varepsilon_{t}^{\tilde{y}}
$$

Suponiendo nuevamente un equilibrio discrecional:

$$
i_{t}=\delta^{-1} A \tilde{\pi}_{t}+\delta^{-1} \theta e_{t}+\delta^{-1} \varepsilon_{t}^{\tilde{y}}
$$

La brecha de inflación y el shock de la brecha del producto tienen el signo esperado, y los movimientos del tipo de cambio real se asocian correctamente a la tasa de política: si la inflación efectiva disminuye por la apreciación cambiaria, la banca central puede mantener una laxa tasa de interés, sin que ello implique la desatención de su meta de inflación; y una depreciación cambiaria que aumente el nivel general de precios, obliga a la autoridad central a robustecer su política monetaria. 
Sustituyendo en (12) las formas reducidas de brecha de inflación y tipo de cambio real (ecuaciones A.2 y A.4 del anexo estadístico, respectivamente), se llega a la expresión más depurada de la regla de política:

$$
\begin{gathered}
i_{t}=f_{\tilde{\pi}} \varepsilon_{t}^{\tilde{\pi}}+f_{\tilde{y}} \varepsilon_{t}^{\tilde{y}}+f_{e} \varepsilon_{t}^{e} \\
f_{\tilde{\pi}}=\left[\delta^{-1}\left(A a_{\tilde{\pi}}+\theta c_{\tilde{\pi}}\right)\right] ; f_{\tilde{y}}=\left[\delta^{-1}\left(A a_{\tilde{y}}+\theta c_{\tilde{y}}+1\right)\right] ; f_{e}=\left[\delta^{-1}\left(A a_{e}+\theta c_{e}\right)\right] \\
\forall f_{j}>0
\end{gathered}
$$

Donde $\varepsilon_{t}^{\tilde{\pi}}$ y $\varepsilon_{t}^{\tilde{y}}$ son los shocks tradicionales de una regla tipo Taylor (Ball, 1997; Svensson, 1996; Taylor, 1993, 1998), y $\varepsilon_{t}^{e}$ la innovación del modelo: el manejo de la política monetaria es per se un índice de cotizaciones monetarias (ICMs) que incluye la tasa de interés nominal y tipo de cambio real (Ball, 1998; Svensson, 1998; Taylor, 2001) $)^{3}$.

Aunque la ecuación (13) no muestra las variables del lado derecho de la igualdad en sus valores de estado, al efectuarse la estabilización del producto e inflación por los canales que suscribe la regla de Taylor, la expresión obtenida se considera una regla de política óptima (donde la determinación de los coeficientes $f_{j}$, está sujeta a las preferencias de los policy makers).

\section{EVIDENCIA EMPIRICA}

\subsection{Revisión de la literatura}

Desde su implementación en Nueva Zelanda, la valoración empírica del régimen de metas de inflación ha propiciado un debate internacional, a fin de examinar el rol de la política monetaria dentro de la nueva macroeconomía. En un primer acercamiento, al forjarse un vínculo de confianza entre banca central, gobierno y sector público, la fijación de metas de inflación reduce la tasa promedio y shocks de inflación (Roger y Stone, 2005; Sterne, 2001), además de promover un crecimiento económico con menor variabilidad en el producto (García-Solanes y Torrejón-Flores, 2012; Gonçalves y Salles, 2008). De forma anecdótica, Angeriz y Arestis (2009) señalan que la evidencia más fuerte sobre los buenos resultados de la estrategia, consiste en que los países que la siguen generalmente están complacidos con ella, pues hasta el momento, ninguno

3 En el primer apartado del anexo estadístico se determinan los valores de $a_{j}$ y $c_{j}$ de la ecuación (13). 
de ellos la ha abandonado (para una compilación al respecto, véase Hammond, 2012 y Schmidt-Hebbel y Carrasco, 2016).

En un escenario menos alentador, al analizar Cecchetti y Ehrmann (1999) las preferencias de política de 23 economías ( 9 con metas de inflación y 14 sin ellas), no encuentran diferencias relevantes en los coeficientes de aversión a la inflación; ipso facto: los buenos resultados del esquema son los propios de un período "friendly to increased price stability" (Neumann y von Hagen, 2002: 129). Por el contrario, de Paula y Ferrari-Filho (2010) dan dos explicaciones de por qué dicha estrategia tiene un menor éxito en las economías emergentes: (i) como sus canales de crédito están muy poco desarrollados, la demanda agregada es igual de (in)sensible a los mecanismos de transmisión de la política monetaria; y (ii) como su gasto en consumo no es del todo significativo frente al efecto riqueza, la tasa de interés no tiene repercusiones de importancia en el consumo de la gente.

Con la disponibilidad de estadísticas más extensas, diversas investigaciones han estudiado el comportamiento de la banca central a través del análisis econométrico de las reglas de política. Por ejemplo, al estimar Corbo, Landerretche y Schmidt-Hebbel (2001) la regla de Taylor para 25 economías (Mínimos Cuadrados Ordinarios, MCO), prueban que únicamente en Australia y Colombia (con metas de inflación) y Estados Unidos y los Países Bajos (sin metas de inflación), la tasa de interés rezagada, brecha de inflación y brecha del producto tienen coeficientes positivos y estadísticamente significativos. En la Unión Europea, Gerdesmeier y Roffia (2003) y Gerlach y Schnabel (2000) obtienen en la mayoría de sus regresiones (Método de los Momentos Generalizados, MMG) coeficientes de la brecha de inflación superiores al de la brecha del producto, lo que presupone un mayor compromiso por la estabilidad de precios que la actividad económica de la zona.

En el caso particular de las economías emergentes, la mayor sensibilidad a los movimientos del tipo de cambio, requiere que las reglas de política sean las de una economía abierta. Con Schmidt-Hebbel y Werner (2002), los coeficientes (MCO) de la regla de Taylor para Brasil, Chile y México, muestran que sus tipos de cambio nominales no tienen ningún efecto significativo sobre la tasa de interés; aun cuando sus esterilizaciones cambiarias dan pie al fenómeno documentado como "miedo a flotar" (Calvo y Reinhart, 2000). En contrapartida, al realizar de Mello y Moccero (2011) modelos de Vectores Autorregresivos Estructurales (SVAR) para Brasil, Chile, Colombia y México, concluyen que únicamente en el último la tasa de política responde significativamente a las variaciones del tipo de cambio nominal; sin importar que durante la segunda mitad del período analizado, el esquema monetario corresponde (enteramente) al de metas de inflación.

Entre los trabajos que analizan exclusivamente la economía mexicana, se encuentran Ramos-Francia y Torres (2005) y Cermeño, Villagómez y Orellana 
Polo (2012). En el primero, al estimar en seis ocasiones una regla de política con modelos de Vectores Autorregresivos (VAR): la meta de inflación y brecha de sus expectativas siempre son significativas y mayores que la unidad, la brecha del producto solo es relevante en dos ocasiones (y menor que 1), y la depreciación cambiaria peso-dólar en cuatro (con coeficientes que oscilan entre 0,67-0,84). Con Cermeño, Villagómez y Orellana Polo (2012), al analizar por MMG el período 1998-2008, los coeficientes de la brecha del producto y desviación esperada del tipo de cambio real son significativos pero menores que la unidad ( 0,63 y 0,23 , respectivamente), mientras que la meta de inflación y brecha de sus expectativas tienen nuevamente los valores más altos $(1,15$ y 1,18$)$.

Salvo algunos matices en la determinación de los objetivos de política, la evidencia presentada (particularmente en los modelos autorregresivos) supone la manipulación deliberada de la trayectoria del tipo de cambio en México durante el esquema de metas de inflación: "the case for using two policy instruments (the policy interest rate and FX market intervention) under an IT [inflation targeting] regime" (Ghosh, Ostry y Chamon, 2016: 4). En este sentido, más que una flotación intervenida o "miedo a flotar", algunos autores consideran el caso de México como "miedo a depreciar": intervención asimétrica del mercado cambiario (Libman, 2018), combatiendo con mayor intensidad las depreciaciones del mismo (Barbosa-Filho, 2015; Ros, 2015). Esto último, difiere notablemente de la experiencia mercantilista del este y sureste de Asia (Pontines y Siregar, 2012, 2015), donde el "miedo a apreciar" o "flotación a la inversa" (Levy-Yeyati y Sturzenegger, 2007; Levy-Yeyati, Sturzenegger y Gluzmann, 2013) permite conservar un tipo de cambio competitivo en protección de los sectores exportables (Guzmán, Ocampo y Stiglitz, 2017; LevyYeyati y Sturzenegger, 2009).

\subsection{Estimaciones econométricas}

Tomando como marco de referencia la regla de política de la segunda sección y la revisión de la literatura previamente descrita, en este apartado se estima bajo la metodología de un modelo SVAR cointegrado, "el rol del tipo de cambio real en México durante el esquema de metas de inflación". Para la construcción de la base de datos, exceptuando el tipo de cambio real obtenido del Banco de México (BANXICO, 2017), la tasa de interés nominal, el Indice Nacional de Precios al Consumidor (INPC), y el Indice Global de la Actividad Económica (IGAE) provienen del Instituto Nacional de Estadística y Geografía (INEGI, 2017)4. Por su parte, la elaboración de las

4 El tipo de cambio real es un índice ponderado con los precios al consumidor de 111 economías, y la tasa de interés nominal el promedio ponderado de instrumentos de deuda de corto plazo a 28 días. 
brechas de inflación y producto requieren que se satisfagan las siguientes ecuaciones: $\tilde{\pi}_{t}=(I N P C-M I)$ y $\tilde{y}_{t}=\left(I G A E-T^{H P}\right)$, donde MI es la meta de inflación y $\mathrm{T}^{\mathrm{HP}}$ la tendencia del producto generada con el filtro Hodrick-Prescott (HP).

Aunque el inicio y consolidación de las metas de inflación en el país datan de 1999 y 2002, respectivamente (véase más adelante Figura 1), después de realizarse diversas estimaciones a partir de diferentes lapsos de tiempo, el período de estudio a considerar por la significancia de sus resultados empieza en octubre de 2003 y termina en enero de 2015: $t=2003$ M10 - 2015M01. En lo tocante a la frecuencia de las series, como la producción económica es la única variable disponible mensual y trimestralmente, se ha preferido optar por la primera (el IGAE), para así preservar en el resto de la información estadística su estado original (además que un mayor número de observaciones tiene la particularidad de generar estimaciones más consistentes).

De esta manera, la expresión matemática de la ecuación (13) en su estructura de modelo VAR, se plantea como sigue:

$$
\begin{gathered}
x_{t}=H_{1} x_{t-1}+H_{2} x_{t-2}+\cdots+H_{p} x_{t-p}+K z_{t}+\epsilon_{t} \\
x_{t}=\left[i_{t}, \tilde{y}_{t}, e_{t}, \tilde{\pi}_{t}\right]
\end{gathered}
$$

Donde $x_{t}$ es un vector de $m$ variables endógenas (ordenadas de izquierda a derecha por su mayor endogeneidad), $z_{t}$ un vector de $n$ variables exógenas, $H$ y $K$ las matrices de los coeficientes a estimar, y $\epsilon_{t}$ el vector de innovaciones no correlacionado con sus rezagos ni las demás variables. Como los retardos de $x_{t}$ únicamente aparecen a un lado de la igualdad, la estimación por MCO no deriva en problemas de simultaneidad (Wooldridge, 2016: cap. 16); por lo que sus estimadores además de eficientes son equivalente a los de Mínimos Cuadrados Generalizados (MCG) (EViews, 2015: cap. 38).

Para llevar a cabo el análisis de cointegración propuesto, lo primero a realizar supone la determinación de la estacionariedad de las variables, es decir, que sus distribuciones de probabilidad no dependan del tiempo (Suriñach et al., 1995: cap. 2). Para ello, al aplicarse en las cuatro series involucradas la prueba de raíz unitaria de la Tabla 1, se determina al $95 \%$ de confianza la estacionariedad de las variables en su primera diferencia: series $I$ (1) o integrables de orden 1. En presencia de procesos con raíz unitaria, la inferencia clásica del VAR se plantea a través de un modelo de Vector de Corrección del Error (VEC), dejando para su correspondiente elaboración las tres siguientes etapas: (i) selección del número de rezagos óptimos; (ii) construcción del polinomio determinístico del modelo; y (iii) determinación del rango de cointegración (Johansen, 1995). 


\section{TABLA 1}

\section{PRUEBA KPSS}

\begin{tabular}{|c|c|c|c|c|c|c|c|}
\hline$I(0)$ & Modelo & $t_{\text {prueba }}$ & $t_{0,05}$ & $I(1)$ & Modelo & $t_{\text {prueba }}$ & $t_{0,05}$ \\
\hline \multirow{2}{*}{$i_{t}$} & I y T & 0,21 & 0,15 & $\Delta i_{t}$ & I y T & 0,07 & 0,15 \\
& I & 2,00 & 0,46 & & I & 0,18 & 0,46 \\
\hline \multirow{2}{*}{$\tilde{y}_{t}$} & I y T & 0,50 & 0,15 & $\Delta \tilde{y}_{t}$ & I y T & 0,04 & 0,15 \\
& I & 0,52 & 0,46 & & I & 0,05 & 0,46 \\
\hline \multirow{2}{*}{$e_{t}$} & I y T & 5,70 & 0,15 & $\Delta e_{t}$ & I y T & 0,00 & 0,15 \\
& I & 19,68 & 0,46 & & I & 0,00 & 0,46 \\
\hline \multirow{2}{*}{$\tilde{\pi}_{t}$} & I y T & 3,29 & 0,15 & $\Delta \tilde{\pi}_{t}$ & I y T & 0,00 & 0,15 \\
& I & 5,48 & 0,46 & & I & 0,00 & 0,46 \\
\hline
\end{tabular}

$\Delta=$ Primera diferencia.

$\mathrm{I}=$ Intercepto; $\mathrm{T}=$ Tendencia lineal.

$\mathrm{H}_{0}=$ Raíz unitaria; $\mathrm{H}_{\mathrm{a}}=$ Serie estacionaria.

Fuente: Elaboración propia.

En la selección del número de rezagos, por los criterios de información de índice de probabilidad modificado (LR en inglés), error de predicción final (FPE), Akaike, Schwarz y Hannan-Quinn (Lütkepohl, 2005: cap. 4), se establece la existencia de hasta cuatro posibles escenarios para la discriminación de los retardos del modelo (Tabla 2). Al probarse en cada uno de ellos la significancia de sus resultados, el criterio de Akaike arroja los únicos estimadores que además de satisfactorios en su interpretación económica, cumplen con las pruebas de estabilidad requeridas (véase más adelante Tablas A.1, A.2 y A.3 del anexo estadístico).

TABLA 2

SELECCION DE REZAGOS

\begin{tabular}{|c|c|c|c|c|c|}
\hline Rezagos & LR & FPE & Akaike & Schwarz & Hannan-Quinn \\
\hline 1 & - & 0,30 & 10,14 & $10,48^{*}$ & 10,28 \\
2 & 58,29 & 0,24 & 9,92 & 10,61 & $10,20^{*}$ \\
16 & $28,32^{*}$ & $0,11^{*}$ & 8,83 & 14,32 & 11,06 \\
27 & 18,32 & 0,19 & $7,38^{*}$ & 16,63 & 11,14 \\
\hline
\end{tabular}

* Indica el número de rezagos.

Fuente: Elaboración propia. 
En la construcción del polinomio del modelo y determinación del rango de cointegración, por los criterios de información de la Tabla 3 y las pruebas de traza y máximo valor propio de la Tabla 4, se confirma la significancia de al menos tres relaciones de cointegración $\left(\dot{x}_{3}\right)$ con intercepto y tendencia cuadrática en común ( $I$ y $T^{2}$, respectivamente):

$$
x_{4, t}=\vartheta \dot{x}_{3, t}
$$

Resolviendo para cada variable:

$$
\begin{gathered}
\Delta i_{1, t}=\varphi_{1}\left(x_{4, t}-\vartheta \dot{x}_{3, t}-I-T^{2}\right)+\epsilon_{1, t} \\
\Delta \tilde{y}_{2, t}=\varphi_{2}\left(x_{4, t}-\vartheta \dot{x}_{3, t}-I-T^{2}\right)+\epsilon_{2, t} \\
\Delta e_{3, t}=\varphi_{3}\left(x_{4, t}-\vartheta \dot{x}_{3, t}-I-T^{2}\right)+\epsilon_{3, t} \\
\Delta \tilde{\pi}_{4, t}=\varphi_{4}\left(x_{4, t}-\vartheta \dot{x}_{3, t}-I-T^{2}\right)+\epsilon_{4, t} \\
0>\varphi_{j}<-1
\end{gathered}
$$

Donde los $\varphi_{j}$ correctores del error tienden a cero, conforme las relaciones a largo plazo de las cuatro variables endógenas se ajustan parcialmente.

\section{TABLA 3}

POLINOMIO DEL MODELO

\begin{tabular}{|c|c|c|c|c|c|c|c|c|c|c|}
\hline \multirow{3}{*}{$\begin{array}{c}\text { Ecn(s). de } \\
\text { cointegración }\end{array}$} & \multicolumn{5}{|c|}{ Akaike } & \multicolumn{5}{|c|}{ Schwarz } \\
\cline { 2 - 11 } & - & $\mathrm{I}$ & $\mathrm{I}$ & $\mathrm{I}$ & $\mathrm{I}$ & - & $\mathrm{I}$ & $\mathrm{I}$ & $\mathrm{I}$ & $\mathrm{I}$ \\
\cline { 2 - 11 } & - & - & - & $\mathrm{T}$ & $\mathrm{T}^{2}$ & - & - & - & $\mathrm{T}$ & $\mathrm{T}^{2}$ \\
\hline 0 & 7,61 & 7,61 & 7,58 & 7,58 & 7,39 & 16,86 & 16,86 & 16,92 & 16,92 & 16,82 \\
1 & 7,10 & 7,08 & 7,06 & 6,27 & 6,06 & 16,52 & 16,53 & 16,57 & 15,80 & 15,66 \\
2 & 6,96 & 6,95 & 6,93 & 5,77 & 5,61 & 16,55 & 16,59 & 16,61 & 15,49 & 15,37 \\
3 & 6,92 & 6,93 & 6,92 & 5,67 & $5,56^{*}$ & 16,68 & 16,76 & 16,77 & 15,59 & $15,50^{*}$ \\
\hline
\end{tabular}

* Indica el tipo de modelo.

Fuente: Elaboración propia. 
TABLA 4

RANGO DE COINTEGRACION

\begin{tabular}{|c|r|r|r|r|}
\hline \multirow{2}{*}{ Rango } & \multicolumn{2}{|c|}{ Traza } & \multicolumn{2}{c|}{ Máx. valor propio } \\
\cline { 2 - 5 } & $t_{\text {prueba }}$ & $t_{0,05}$ & $t_{\text {prueba }}$ & $t_{0,05}$ \\
\hline 0 & 297,26 & 55,25 & 196,59 & 30,82 \\
1 & 100,66 & 35,01 & 78,08 & 24,25 \\
2 & 22,58 & 18,40 & 22,54 & 17,15 \\
3 & 0,04 & 3,84 & 0,04 & 3,84 \\
\hline
\end{tabular}

$\mathrm{H}_{0}: t_{\text {prueba }}<t_{0,05}$.

Fuente: Elaboración propia.

Especificada la estructura del modelo, siendo los residuales del primer vector los únicos que cumplen las hipótesis básicas de MCO (Tablas A.1, A.2 y A.3), el sistema de información de la ecuación (16) se reduce al mismo número de relaciones cointegrantes:

$$
\begin{array}{cc}
\Delta \dot{i}_{1, t}= & \varphi_{1}\left(x_{4, t}-\vartheta \dot{x}_{1, t}-I-T^{2}\right)+\epsilon_{1, t} \\
\vdots & \vdots \\
\Delta \tilde{\pi}_{4, t}= & \varphi_{4}\left(x_{4, t}-\vartheta \dot{x}_{1, t}-I-T^{2}\right)+\epsilon_{4, t}
\end{array}
$$

Como el corrector del error de la tasa de interés $\left(\varphi_{1}\right)$ es el único parámetro de ajuste que además de significativo tiene el valor esperado (Tabla 5), la normalización de los resultados es la siguiente:

$$
\Delta i_{t-1}=0.26-0.30\left(i_{t-1}\right)+0.07\left(\tilde{y}_{t-1}\right)+0.04\left(e_{t-1}\right)+0.38\left(\tilde{\pi}_{t-1}\right)-0.01\left(T^{2}\right)
$$

Donde un incremento del $1 \%$ en el tipo de cambio real y brecha de inflación, suponen un crecimiento del 0,04 y $0,38 \%$ de la tasa de interés nominal, respectivamente; y la brecha del producto (por su parámetro $t$ ), no tiene una significancia estadística en la determinación de la política monetaria.

En cuanto a los demás resultados, el valor del intercepto en la ecuación (18) refleja la tasa de interés real de la economía $(0,26)$, la tendencia cuadrática el crecimiento de $\Delta i_{t-1}$ a través del tiempo $(-0,01)$, y el parámetro de ajuste el tiempo que le toma 
a la tasa de interés nominal (ante un shock externo) regresar a su estado de equilibrio (0,30\% de mes).

TABLA 5

MODELO VEC

\begin{tabular}{|c|c|c|c|}
\hline \multicolumn{2}{|c|}{ Ecuación de cointegración } & \multicolumn{2}{|c|}{ Parámetros de ajuste } \\
\hline \multirow{3}{*}{$i_{t-1}$} & 1,00 & $\varphi_{1}$ & $\begin{array}{c}-0,30 \\
(0,16) \\
{[-1,84]}\end{array}$ \\
\hline \multirow{2}{*}{$\tilde{y}_{t-1}$} & $-0,23$ & & 2,57 \\
& $(0,25)$ & $\varphi_{2}$ & $(0,78)$ \\
& {$[-0,92]$} & & {$[3,27]$} \\
\hline \multirow{3}{*}{$e_{t-1}$} & $-0,12$ & & 1,77 \\
& $(0,02)$ & $\varphi_{3}$ & {$[1,32)$} \\
\hline \multirow{2}{*}{$\tilde{\pi}_{t-1}$} & {$[-7,66]$} & & 0,05 \\
& $-1,27$ & & $(0,16)$ \\
\hline$I$ & {$[0,21)$} & $\varphi_{4}$ & \\
\hline$T^{2}$ & $-0,6,09]$ & & \\
\hline
\end{tabular}

Error estándar: ( ).

Estadístico t: [ ].

$\left|t_{0,10 \%}\right| \leq 1,66 ;\left|t_{0,05 \%}\right| \leq 1,98 ;\left|t_{0,01 \%}\right| \leq 2,63$.

Fuente: Elaboración propia.

En su análisis estructural, al ortogonalizar por descomposición de Cholesky la varianza de $i_{t}$ en los shocks del vector de variables endógenas (ecuación 14), se obtienen los porcentajes que cada una de ellas explican la tasa de política (Tabla 6). Durante los primeros seis meses, la varianza de la tasa de interés es la variable de mayor importancia en la determinación de su propio comportamiento $(72,64 \%)$, seguida de la brecha de inflación (14,66\%), el tipo de cambio real $(9,46 \%)$, y finalmente la brecha del producto $(3,24 \%)$. Del séptimo mes en adelante, la brecha de inflación y tipo de cambio real intercambian de posición, y la tasa de interés nominal y brecha del producto se mantienen igual. 
TABLA 6

VARIANZA DE LA TASA DE INTERES NOMINAL (\%)

\begin{tabular}{|c|c|c|c|c|c|}
\hline Meses & $\begin{array}{c}\text { Errores } \\
\text { estándar }\end{array}$ & $i_{t}$ & $\tilde{y}_{t}$ & $e_{t}$ & $\tilde{\pi}_{t}$ \\
\hline 1 & 0,26 & 100,00 & 0,00 & 0,00 & 0,00 \\
2 & 0,42 & 92,39 & 0,79 & 0,08 & 6,74 \\
3 & 0,57 & 88,63 & 0,50 & 1,04 & 9,83 \\
4 & 0,73 & 80,54 & 0,39 & 3,42 & 15,65 \\
5 & 0,90 & 75,26 & 1,83 & 7,15 & 15,75 \\
6 & 1,14 & 72,64 & 3,24 & 9,46 & 14,66 \\
7 & 1,37 & 68,54 & 5,50 & 12,33 & 13,62 \\
8 & 1,60 & 64,22 & 8,79 & 15,23 & 11,76 \\
9 & 1,80 & 62,64 & 9,88 & 16,65 & 10,83 \\
\hline
\end{tabular}

Fuente: Elaboración propia.

En este contexto, los resultados obtenidos tanto por el análisis de cointegración (largo plazo) como por el de descomposición de la varianza (simulación de corto plazo), son consistentes con una política monetaria que además de priorizar la estabilidad de precios, se mantiene inflexible a través del tiempo; es decir, la función de reacción del Banco de México, supone un anclaje intertemporal de las metas de inflación, con la tasa de interés nominal y tipo de cambio real como sus instrumentos de trabajo.

\subsection{Evidencia estadística}

Para tener una mejor perspectiva de la evidencia econométrica, a continuación se compara información estadística de las metas de inflación en México, así como del resto de economías en América Latina que siguen la estrategia ${ }^{5}$. Empezando con los intervalos de inflación (Figura 1), Brasil y Guatemala tienen los rangos más flexibles, Chile, Colombia y México están en la media de la muestra, y Perú es la economía que permite la menor discrecionalidad. Si se contabilizan los años que cada país ha mantenido su nivel general de precios en los intervalos deseados (marcados con un punto negro), Brasil y Colombia obtienen los mejores resultados (superiores al 60\%), Chile, Guatemala y Perú (el menos flexible) tienen una efectividad que sobrepasa el $50 \%$, y México es la economía con los peores resultados (45\%).

5 Al compartir el esquema de metas de inflación, los países de la muestra utilizan la tasa de interés nominal como su instrumento de política monetaria, los tipos de cambio son de libre flotación, y sus bancas centrales autónomas. Esto último difiere de Brasil, aunque el comportamiento de su autoridad monetaria es similar al de sus homólogos (Pérez-Laurrabaquio, 2019). 


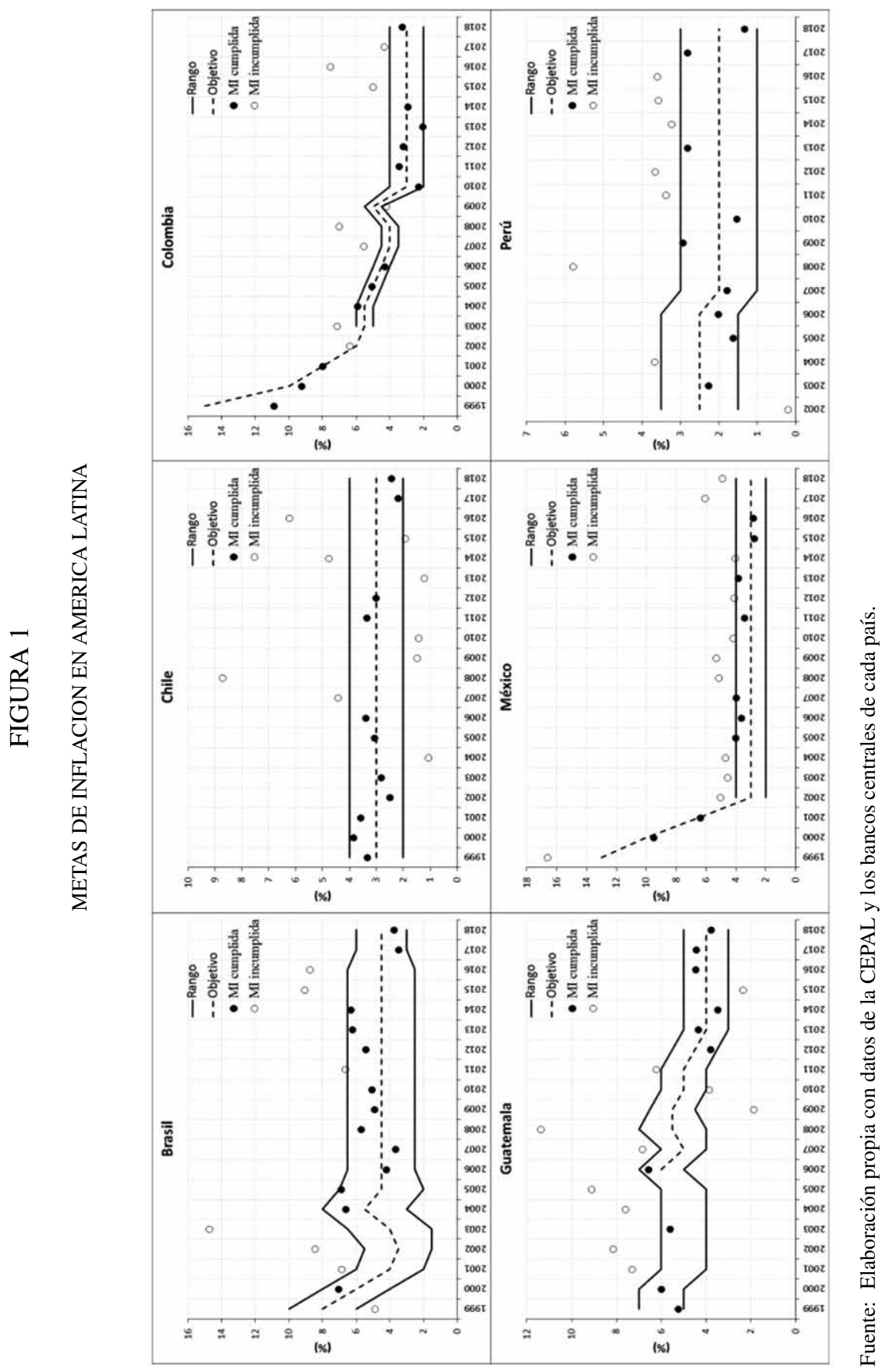


En la fijación de las tasas de política hacia el oportuno cumplimiento de las metas de inflación (Figura 2), Brasil es el único país que en todo momento mantiene su tasa de interés por encima de la inflación general, México no logra hacerlo en 2014, y los demás países en más de dos ocasiones ${ }^{6}$. Incluyendo en el análisis las tasas de crecimiento económico (Figura 3), Chile, Colombia, Guatemala y Perú tienen los mejores resultados (superiores al 3\%), Brasil se halla en el penúltimo sitio $(2,4 \%)$, y finalmente se encuentra México (2,23\%). En este sentido, pese a que economías como la brasileña mantienen una estricta política monetaria, la tasa de crecimiento de su PIB supera lo mostrado por países como México, donde una tasa de interés relativamente estable y descendiente no tiene los resultados esperados en el estímulo de su actividad económica.

Retomando el análisis de economías abiertas, al contabilizar el número de años que cada país ha mantenido su tipo de cambio real apreciado (Figura 4), Guatemala se encuentra en la cima con trece de diecisiete ocasiones, le sigue Brasil con nueve, Chile, Perú y México con siete, y Colombia con seis (todos ellos marcados con un punto negro). En el caso que nos compete, aunque la variación del tipo de cambio en México es la única que mantiene una trayectoria depreciativa a lo largo de la muestra (línea negra punteada), sus escasas apreciaciones cambiarias tienen una razonable incidencia en el cumplimiento de las metas de inflación: del total de años que se ha apreciado su tipo de cambio, el 57\% de ellos obtienen los niveles deseados (marcados con MI). Por consiguiente, las políticas monetaria y cambiaria de México conservan una regularidad de importancia: el cumplimiento de las metas de inflación es consistente con la apreciación del tipo de cambio; en otras palabras: el tipo de cambio real en México se manifiesta (nuevamente) como un instrumento intermedio del banco central.

\section{CONCLUSIONES}

En la contribución del análisis de economías abiertas con imperfecta movilidad de capitales, en este trabajo se investigó "el rol del tipo de cambio real en México durante el esquema de metas de inflación”. Tomando como marco de referencia la política monetaria de la Nueva Macroeconomía Keynesiana, en la sección 2 se construyó una regla monetaria de tasa de interés, propia de una economía como la mexicana: pequeña, abierta, con tipo de cambio intervenido (de facto) y banca central independiente. Entre sus particularidades, la función de reacción incorpora la brecha de inflación y brecha del producto como sus objetivos de política monetaria, y la tasa de interés nominal y tipo de cambio real como sus instrumentos de trabajo.

6 La importancia de mantener la tasa de política por encima del nivel general de precios, radica en que un adecuado manejo de la misma, supone que las presiones de inflación se eliminen incrementando más que proporcionalmente la tasa de interés nominal (Taylor, 1993). 


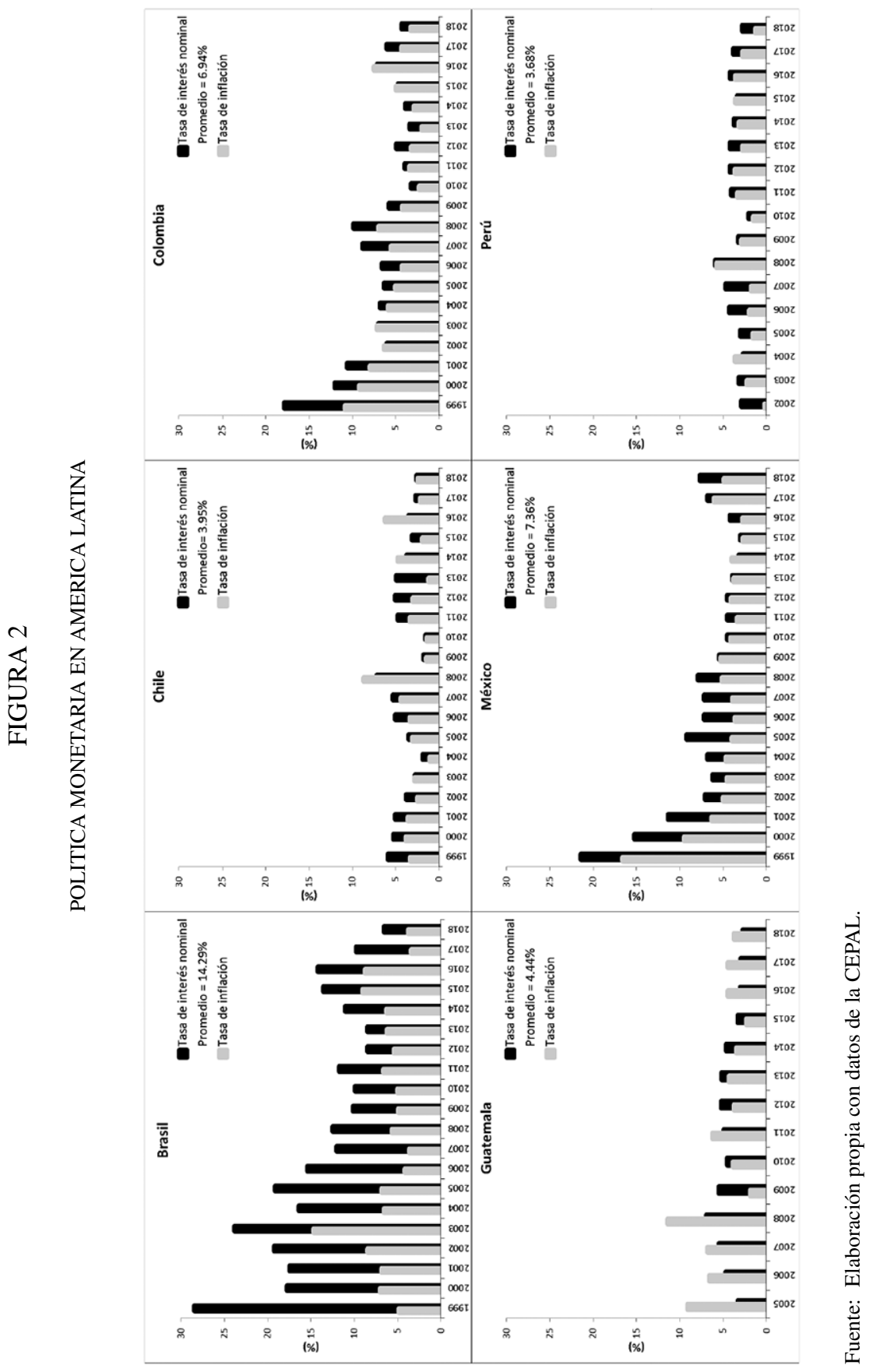




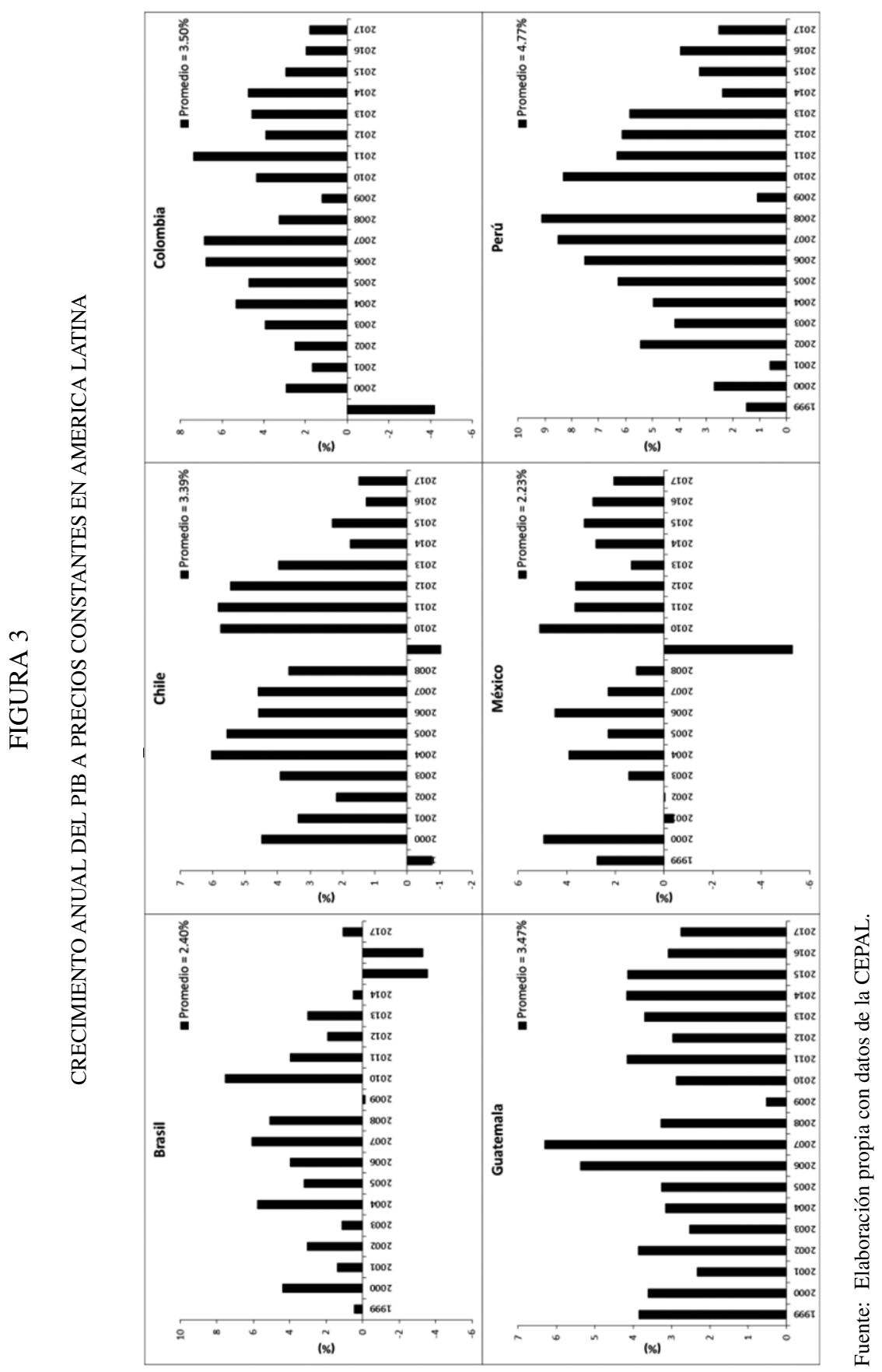




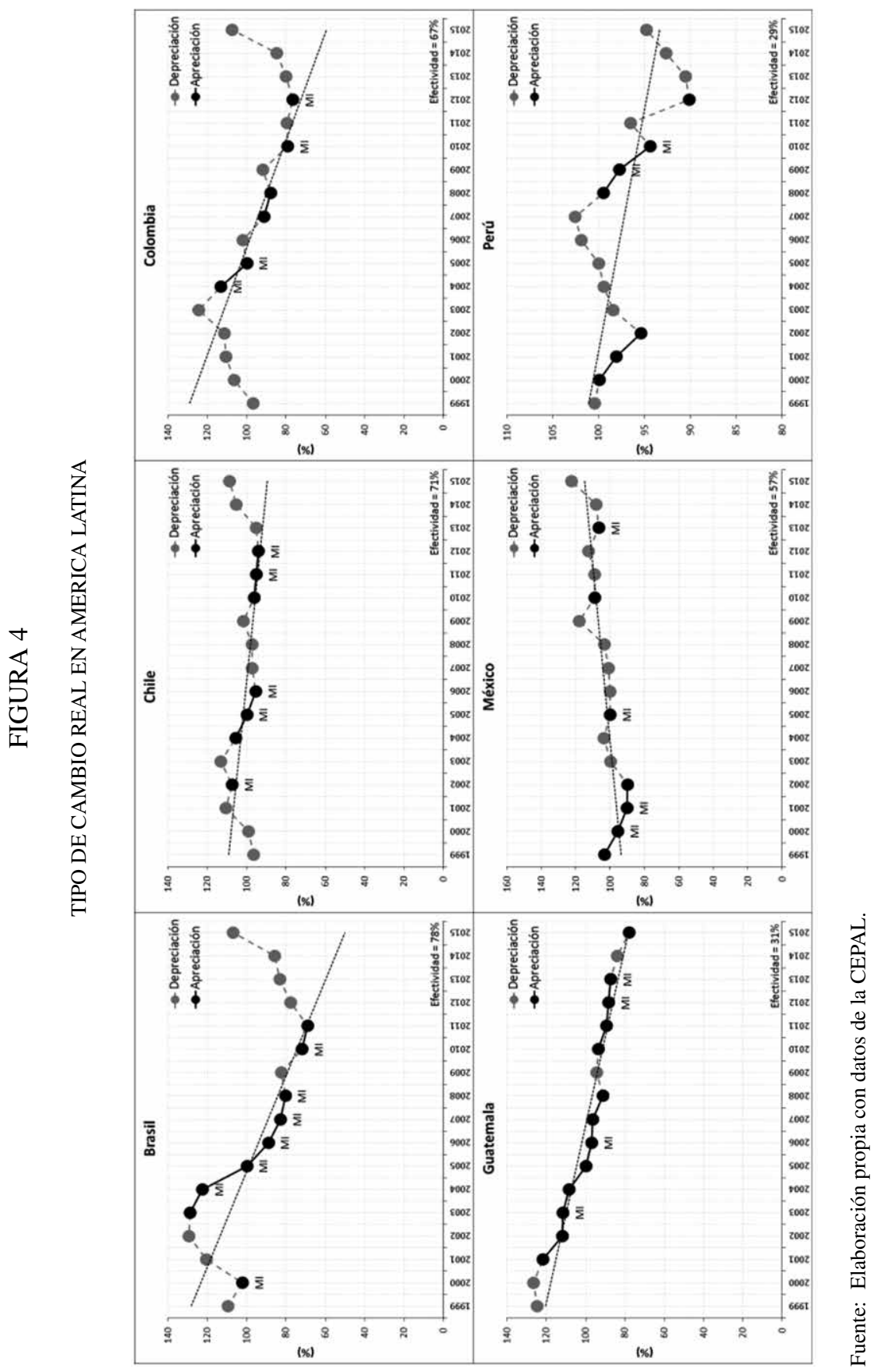


Para su tratamiento empírico, con la selección de los modelos autorregresivos como el mecanismo de validación más apropiado en la estimación de las reglas de política, en la sección 3 se estimó un Vector de Corrección del Error para el período 2003M10-2015M01. En su análisis de cointegración (Tabla 5), la tasa de interés nominal responde significativamente a las variaciones de brecha de inflación y tipo de cambio real, mientras que la brecha del producto no tiene una significancia estadística en la determinación de la política monetaria. En su análisis de descomposición de la varianza (Tabla 6), la brecha de inflación y tipo de cambio real son las variables de mayor importancia en la explicación de la tasa de interés, y en última instancia la brecha del producto.

En relación con la evidencia estadística (1999-2018), al compararse el funcionamiento de las metas de inflación en el país con el resto de economías en América Latina que siguen la estrategia, se encuentra lo siguiente: (i) el éxito de las metas de inflación en México se asume como moderado (del 45\%), ya que, de todos los países de la muestra, tiene una mayor efectividad en el cumplimiento de la estrategia (Figura 1); (ii) con una tasa de interés relativamente estable y descendiente (Figura 2), la política monetaria de México no tiene los resultados esperados en el estímulo de su actividad económica (Figura 3), lo que sí ocurre en economías como la brasileña, a pesar de mantener una estricta política monetaria; y (iii) con una sobresaliente depreciación del tipo de cambio (Figura 4), las escasas apreciaciones en México tienen una de las mayores incidencias en el cumplimiento de las metas de inflación.

Por consiguiente, tanto en su enfoque econométrico como estadístico, la evidencia presentada describe al régimen de metas de inflación en el país como un sistema híbrido, donde la tasa de interés nominal (instrumento de política) y apreciaciones del tipo de cambio real (instrumento intermedio), tienen como primordial objetivo, la estabilización de la inflación dentro de una meta numérica.

\section{BIBLIOGRAFIA}

1. ANGERIZ, A.; Arestis, P. (2009). "Objetivo de inflación: evaluación de la evidencia”. Investigación Económica, 68 (SPE.), 21-46.

2. ARESTIS, P.; Sawyer, M.C. (2003). "Inflation targeting: a critical appraisal". Levy Economics Institute, Working Paper 388.

3. BALL, L.M. (1997). "Efficient rules for monetary policy". National Bureau of Economic Research (NBER), Working Paper 5952.

4. BALL, L.M. (1998). "Policy rules for open economies". NBER, Working Paper 6760.

5. BANXICO. (2017). "Indice de tipo de cambio real", http://www.banxico.org.mx/SieInternet/ consultarDirectorioInternetAction.do?sector=6\&accion=consultarCuadro\&idCuadro=CR60\&local $\mathrm{e}=\mathrm{es}$ [Accedido Diciembre 25, 2017].

6. BARBOSA-FILHO, N.H. (2015). "Monetary policy with a volatile exchange rate: the case of Brazil since 1999". Comparative Economic Studies, 57 (3), 401-425.

7. BERNANKE, B.S.; Mishkin, F.S. (1997). "Inflation targeting: a new framework for monetary policy?". NBER, Working Paper 5893.

8. BLINDER, A.S. (1999). El banco central: teoría y práctica. Antoni Bosch editor, Barcelona. 
9. CALVO, G.A. (1983). "Staggered prices in a utility-maximizing framework". Journal of Monetary Economics, 12 (3), 383-398.

10. CALVO, G.A.; Reinhart, C.M. (2000). "Fear of floating". NBER, Working Paper 7993.

11. CAMARA DE DIPUTADOS. (1993) [1917]. Constitución Política de los Estados Unidos Mexicanos, http://www.diputados.gob.mx/LeyesBiblio/ref/cpeum.htm [Accedido Junio 30, 2019].

12. CECHETTI, S.G.; Ehrmann, M. (1999). "Does inflation targeting increase output volatility? An international comparison of policymakers' preferences and outcomes". NBER, Working Paper 7426.

13. CEPAL (Comisión Económica para América Latina y el Caribe). (2019). "Estadísticas e Indicadores", http://estadisticas.cepal.org/cepalstat/WEB_CEPALSTAT/estadisticasIndicadores.asp?idioma = e [Accedido Junio 30, 2019].

14. CERMEÑO, R.; Villagómez, F.A.; Orellana Polo, J. (2012). "Monetary policy rules in a small open economy: an application to Mexico". Journal of Applied Economics, 15 (2), 259-286.

15. CLARIDA, R.H.; Galí, J.; Gertler, M. (1999). "The science of monetary policy: a new Keynesian perspective". NBER, Working Paper 7147.

16. CLARIDA, R.H.; Galí, J.; Gertler, M. (2001). "Optimal monetary policy in closed versus open economies: an integrated approach". NBER, Working Paper 8604.

17. CORBO, V.; Landerretche, O.; Schmidt-Hebbel, K. (2001). "Assessing inflation targeting after a decade of world experience". International Journal of Finance \& Economics, 6 (4), 343-368.

18. DE MELLO, L.; Moccero, D. (2011). "Monetary policy and macroeconomic stability in Latin America: the cases of Brazil, Chile, Colombia and Mexico". Journal of International Money and Finance, 30 (1), 229-245.

19. DE PAULA, L.F.; Ferrari-Filho, F. (2010). "Arestis and Sawyer's criticism on the New Consensus Macroeconomics: some issues related to emerging countries". En Fontana, G.; McCombie, J., y Sawyer, M.C. (Eds.), Macroeconomics, finance and money: essays in honour of Philip Arestis. Ed. Palgrave Macmillan, Londres, pp. 19-34.

20. EVIEWS. (2015). EViews 9 user's guide II. IHS Global Inc, Irvine.

21. GARCIA-SOLANES, J.; Torrejón-Flores, F. (2012). "La fijación de metas de inflación da buenos resultados en América Latina". Revista CEPAL, (106), 37-55.

22. GERDESMEIER, D.; Roffia, B. (2003). "Empirical estimates of reaction functions for the euro area". Banco Central Europeo, Working Paper Series 206.

23. GERLACH, S.; Schnabel, G. (2000). "The Taylor rule and interest rates in the EMU area". Economics Letters, 67 (2), 165-171.

24. GHOSH, A.R.; Ostry, J.D.; Chamon, M. (2016). "Two targets, two instruments: monetary and exchange rate policies in emerging market economies". Journal of International Money and Finance, 60, 172-196.

25. GONÇALVES, C.E.S.; Salles, J.M. (2008). "Inflation targeting in emerging economies: what do the data say?". Journal of Development Economics, 85 (1-2), 312-318.

26. GUZMAN, M.M.; Ocampo, J.A.; Stiglitz, J.E. (2017). "Real exchange rate policies for economic development". NBER, Working Paper 23868.

27. HAMMOND, G.M.S. (2012). "State of the art of inflation targeting". Banco de Inglaterra, Handbooks 29.

28. INEGI. (2017). "Banco de Información Económica", https://www.inegi.org.mx/sistemas/bie/ [Accedido Diciembre 25, 2017].

29. JOHANSEN, S. (1995). Likelihood-based inference in cointegrated vector autoregressive models. Oxford University Press, Oxford.

30. LEITEMO, K.; Söderström, U. (2008). "Robust monetary policy in a small open economy". Journal of Economic Dynamics and Control, 32 (10), 3218-3252.

31. LEVY-YEYATI, E.; Sturzenegger, F. (2007). Fear of floating in reverse: exchange rate policy in the 2000. LACEA-LAMES Annual Meetings.

32. LEVY-YEYATI, E.; Sturzenegger, F. (2009). (The effect of) monetary and exchange rate policies (on development). Escuela de Negocios-Universidad Torcuato Di Tella, Documento de Trabajo 03/2009.

33. LEVY-YEYATI, E.; Sturzenegger, F.; Gluzmann, P.A. (2013). "Fear of appreciation". Journal of Development Economics, 101, 233-247. 
34. LIBMAN, E. (2018). "Política monetaria y cambiaria asimétrica en países latinoamericanos que usan metas de inflación". Revista CEPAL, (125), 29-46.

35. LÜTKEPOHL, H. (2005). New introduction to multiple time series analysis. Springer, Berlín.

36. MISHKIN, F.S. (2000). "Inflation targeting in emerging market countries". NBER, Working Paper 7618.

37. MISHKIN, F.S. (2004). "Can inflation targeting work in emerging market countries?". NBER, Working Paper 10646.

38. NEUMANN, M.J.M.; von Hagen, J. (2002). "Does inflation targeting matter?". Review-Federal Reserve Bank of St. Louis, 84 (4), 127-148.

39. PEREZ-LAURRABAQUIO, O. (2014). "Relación no lineal entre la inflación y crecimiento económico: la experiencia de México". Problemas del Desarrollo, 45 (177), 141-167.

40. PEREZ-LAURRABAQUIO, O. (2019). "Banco de México: 25 años de autonomía". Revista Nexos: Economía y Sociedad, 30 de abril.

41. PONTINES, V.; Siregar, R.Y. (2012). "Exchange rate asymmetry and flexible exchange rates under inflation targeting regimes: evidence from four East and Southeast Asian countries". Review of International Economics, 20 (5), 893-908.

42. PONTINES, V.; Siregar, R.Y. (2015). "Do Asian countries fear appreciation against the renminbi?". En Corbett, J., y Xu, Y. (Eds.) Rebalancing economies in financially integrating East Asia. Ed. Routledge-ERIA Studies in Development Economics. Abingdon y Nueva York, pp. 75-101.

43. RAMOS-FRANCIA, M.; Torres, A. (2005). Reducing inflation through inflation targeting: the Mexican experience. BANXICO, Documento de Trabajo 2005-01.

44. RBNZ (Reserve Bank of New Zealand). (1990). "Policy targets agreement for 1990", https://www. rbnz.govt.nz/monetary-policy/policy-targets-agreements/pta1990-mar [Accedido Junio 30, 2019].

45. ROGER, S.; Stone, M.R. (2005). "On target? The international experience with achieving inflation targets”. Fondo Monetario Internacional (FMI), Working Paper 05/163.

46. ROS, J. (2015). "Central bank policies in Mexico: targets, instruments, and performance". Comparative Economic Studies, 57 (3), 483-510.

47. SAVASTANO, M.A.; Masson, P.R.; Sharma, S. (1997). "The scope for inflation targeting in developing countries". FMI, Working Paper 97/130.

48. SCHMIDT-HEBBEL, K.; Carrasco, M. (2016). "The past and future of inflation targeting: implications for emerging-market and developing economies". En Ghate, C., y Kletzer, K.M. (Eds.). Monetary policy in India: a modern macroeconomic perspective. Ed. Springer, Nueva Delhi, pp. 583-622.

49. SCHMIDT-HEBBEL, K.; Werner, A. (2002). Inflation targeting in Brazil, Chile, and Mexico: performance, credibility, and the exchange rate. Banco Central de Chile, Documento de Trabajo 171.

50. STERNE, G. (2001). Inflation targets in a global context. Banco Central de Chile, Documento de Trabajo 114.

51. SURIÑACH, J.; Artís, M.; López, E.; Sansó, A. (1995). Análisis económico regional: nociones básicas de la teoría de la cointegración. Antoni Bosch editor, Barcelona.

52. SVENSSON, L.E.O. (1996). "Inflation forecast targeting: implementing and monitoring inflation targets". NBER, Working Paper 5797.

53. SVENSSON, L.E.O. (1998). "Open-economy inflation targeting". NBER, Working Paper 6545.

54. SVENSSON, L.E.O. (2001). "Independent review of the operation of monetary policy in New Zealand: report of the Minister of Finance", https://www.rbnz.govt.nz/monetarypolicy/about-monetary-policy/ independent-review-of-the-operation-of-monetary-policy-2 [Accedido Junio 30, 2019].

55. TAYLOR, J.B. (1993). "Discretion versus policy rules in practice". Carnegie-Rochester Conference Series on Public Policy, 39, 195-214.

56. TAYLOR, J.B. (1998). "An historical analysis of monetary policy rules". NBER, Working Paper 6768.

57. TAYLOR, J.B. (2001). "The role of the exchange rate in monetary-policy rules". The American Economic Review, 91 (2), 263-267.

58. WOOLDRIDGE, J.M. (2016). Introductory econometrics: a modern approach. South-Western, Mason. 


\section{ANEXO ESTADISTICO}

\subsection{Regla de política}

Tomando los shocks de $\tilde{\pi}_{t}, \tilde{y}_{t}$ y $e_{t}$ como las variables de estado de las ecuaciones (1), (2) y (3), se forma el siguiente sistema matricial:

$$
\left[\begin{array}{c}
\tilde{\pi}_{t} \\
\tilde{y}_{t} \\
e_{t}
\end{array}\right]=\left[\begin{array}{lll}
a_{\tilde{\pi}} & a_{\tilde{y}} & a_{e} \\
b_{\tilde{\pi}} & b_{\tilde{y}} & b_{e} \\
c_{\tilde{\pi}} & c_{\tilde{y}} & c_{e}
\end{array}\right]\left[\begin{array}{c}
\varepsilon_{t}^{\tilde{\pi}} \\
\varepsilon_{t}^{\tilde{y}} \\
\varepsilon_{t}^{e}
\end{array}\right]
$$

Resolviendo para cada variable:

$$
\begin{aligned}
& \tilde{\pi}_{t}=a_{\tilde{\pi}} \varepsilon_{t}^{\tilde{\pi}}+a_{\tilde{y}} \varepsilon_{t}^{\tilde{y}}+a_{e} \varepsilon_{t}^{e} \\
& \tilde{y}_{t}=b_{\tilde{\pi}} \varepsilon_{t}^{\tilde{\pi}}+b_{\tilde{y}} \varepsilon_{t}^{\tilde{y}}+b_{e} \varepsilon_{t}^{e} \\
& e_{t}=c_{\tilde{\pi}} \varepsilon_{t}^{\tilde{\pi}}+c_{\tilde{y}} \varepsilon_{t}^{\tilde{y}}+c_{e} \varepsilon_{t}^{e}
\end{aligned}
$$

Las expresiones (A.2), (A.3) y (A.4) son las formas reducidas de la brecha de inflación, brecha del producto y tipo de cambio real, respectivamente.

Para definir los valores de $a_{j}$ y $c_{j}$ presentes en la regla de política de la ecuación (13), se sustituye a $\tilde{y}_{t}$ de la ecuación (10) en (1) y a $i_{t}$ de la ecuación (11) en (3):

$$
\begin{gathered}
B \tilde{\pi}_{t}=\alpha E_{t} \tilde{\pi}_{t+1}+\gamma e_{t}+\varepsilon_{t}^{\tilde{\pi}} \\
B \equiv(1+\beta A)>0 \\
D e_{t}=D E_{t} e_{t+1}-C \tilde{\pi}_{t}+C E_{t} \tilde{\pi}_{t+1}+\left(i_{t}^{*}-E_{t} \pi_{t+1}^{*}\right)-\delta^{-1} \varepsilon_{t}^{\tilde{y}}+\varepsilon_{t}^{e} \\
C \equiv\left(\delta^{-1} A\right)>0 ; \quad D \equiv\left(1+\delta^{-1} \theta\right)>0
\end{gathered}
$$

Con la persistencia de los valores futuros y variables del exterior como exógenas:

$$
\begin{gathered}
B \tilde{\pi}_{t}=\gamma e_{t}+\varepsilon_{t}^{\tilde{\pi}} \\
D e_{t}=-C \tilde{\pi}_{t}-\delta^{-1} \varepsilon_{t}^{\tilde{y}}+\varepsilon_{t}^{e}
\end{gathered}
$$


Para eliminar la brecha de inflación y tipo de cambio real de las ecuaciones (A.7) y (A.8), se sustituye en cada una de ellas las expresiones (A.2) y (A.4):

$$
\begin{gathered}
B\left(a_{\tilde{\pi}} \varepsilon_{t}^{\tilde{\pi}}+a_{\tilde{y}} \varepsilon_{t}^{\tilde{y}}+a_{e} \varepsilon_{t}^{e}\right)=\gamma\left(c_{\tilde{\pi}} \varepsilon_{t}^{\tilde{\pi}}+c_{\tilde{y}} \varepsilon_{t}^{\tilde{y}}+c_{e} \varepsilon_{t}^{e}\right)+\varepsilon_{t}^{\tilde{\pi}} \\
D\left(c_{\tilde{\pi}} \varepsilon_{t}^{\tilde{\pi}}+c_{\tilde{y}} \varepsilon_{t}^{\tilde{y}}+c_{e} \varepsilon_{t}^{e}\right)=-C\left(a_{\tilde{\pi}} \varepsilon_{t}^{\tilde{\pi}}+a_{\tilde{y}} \varepsilon_{t}^{\tilde{y}}+a_{e} \varepsilon_{t}^{e}\right)-\delta^{-1} \varepsilon_{t}^{\tilde{y}}+\varepsilon_{t}^{e}
\end{gathered}
$$

Por el método de coeficientes indeterminados, (A.9) y (A.10) se descomponen en (A.11) y (A.12), respectivamente:

$$
\begin{gathered}
a_{\tilde{\pi}}=\frac{\gamma c_{\tilde{\pi}}}{B}+\frac{1}{B} ; \quad a_{\tilde{y}}=\frac{\gamma c_{\tilde{y}}}{B} ; \quad a_{e}=\frac{\gamma c_{e}}{B} \\
c_{\tilde{\pi}}=-\frac{a_{\tilde{\pi}} C}{D} ; \quad c_{\tilde{y}}=-\frac{a_{\tilde{y}} C}{D}-\frac{\delta^{-1}}{D} ; \quad c_{e}=-\frac{a_{e} C}{D}+\frac{1}{D}
\end{gathered}
$$

Combinando ambas expresiones, se obtienen finalmente los valores deseados:

$$
\begin{gathered}
a_{\tilde{\pi}}^{\prime}=\frac{D}{E} ; \quad a_{\tilde{y}}^{\prime}=\frac{\gamma \delta^{-1}}{E} ; \quad a_{e}^{\prime}=\frac{\gamma}{E} \\
E \equiv(\gamma C+B D)>0 \\
c_{\tilde{\pi}}^{\prime}=-\frac{C}{E} ; \quad c_{\tilde{y}}^{\prime}=-\frac{\delta^{-1} B}{E} ; \quad c_{e}^{\prime}=\frac{B}{E}
\end{gathered}
$$

\subsection{Modelo VEC}

TABLA A.1

AUTOCORRELACION

\begin{tabular}{|c|c|c|c|}
\hline Rezagos & Est, LM & Rezagos & Est, LM \\
\hline 1 & 0,55 & 7 & 0,90 \\
2 & 0,65 & 8 & 0,80 \\
3 & 0,72 & 9 & 0,03 \\
4 & 0,07 & 10 & 0,67 \\
5 & 0,17 & 11 & 0,51 \\
6 & 0,18 & 12 & 0,33 \\
\hline
\end{tabular}

$\mathrm{H}_{0}=$ No correlación serial.

Fuente: Elaboración propia. 


\section{TABLA A.2}

\section{NORMALIDAD}

\begin{tabular}{|c|c|}
\hline Residuales & Lütkepohl \\
\hline$\epsilon_{t}^{i}$ & 0,21 \\
$\epsilon_{t}^{\tilde{y}}$ & 0,35 \\
$\epsilon_{t}^{e}$ & 0,08 \\
$\epsilon_{t}^{\tilde{\pi}}$ & 0,30 \\
Conjunta & 0,13 \\
\hline
\end{tabular}

$\mathrm{H}_{0}=$ Normalidad multivariante

Fuente: Elaboración propia.

TABLA A.3

HETEROSCEDASTICIDAD

\begin{tabular}{|c|c|}
\hline Residuales & Wald $\left(\mathrm{chi}^{2}\right)$ \\
\hline$\epsilon_{t}^{i}$ & 0,00 \\
$\epsilon_{t}^{\tilde{y}}$ & 0,00 \\
$\epsilon_{t}^{e}$ & 0,00 \\
$\epsilon_{t}^{\tilde{\pi}}$ & 0,00 \\
\hline
\end{tabular}

$\mathrm{H}_{0}=$ Caminata aleatoria.

Fuente: Elaboración propia. 
